\title{
Psoas haematoma complicating dengue haemorrhagic fever: a case report
}

\author{
AM Ameer ${ }^{1}$, W Kodikara Arachchi ${ }^{2}$, PAJayasingha ${ }^{3}$ \\ ${ }^{1}$ Registrar in Medicine, ${ }^{2}$ Consultant Physician, ${ }^{3}$ Resident Physician, Teaching Hospital Karapitiya, Galle. \\ e-mail address of the corresponding author,Dr.A.M.Ameer: dramameer@gmail.com
}

\section{Introduction}

Dengue fever is a debilitating mosquito-borne disease caused by Dengue virus. It is potentially fatal, particularly to young children and the elderly. Because of its serious emerging health threats, it has aroused considerable medical and public health concerns worldwide. Sri Lanka experienced the biggest outbreak of Dengue fever in 2004 with the highest recorded mortality ${ }^{2}$, but increased incidence of Dengue cases has been noted this year, too (2009). The number of Dengue cases reported in 2009 has gone up to 13,500 and death toll due complications has exceeded 150 during the first six months. Dengue Haemorrhagic Fever (DHF) and Dengue Shock Syndrome (DSS) may manifest in various forms, ranging from petechial skin hemorrhages to life-threatening gastrointestinal bleeding and disseminated intravascular coagulation. We report a case of Psoas haematoma complicating DHF. Such manifestations are not well documented in the literature.

\section{Case report}

A 77 year-old previously well male presented with fever, myalgia and headache of four days duration and generalized weakness of three days duration. Initially, he was admitted to a local hospital. The fever settled on the fourth day but there was no improvement of the generalized weakness and faintishness which developed on the fourth day of the illness. Then the patient was admitted to Teaching Hospital Karapitiya on the sixth day of the illness.

On admission, the patient was mildly dyspnoeic, pale, and had bleeding at venepuncture sites. Pulse rate was $100 \mathrm{bpm}$; blood pressure was $80 / 60 \mathrm{mmHg}$. Lung bases were dull to percussion and breath sounds were reduced on auscultation. Examination of the abdomen revealed generalized tenderness with no guarding or rigidity and there was shifting dullness. Over the course of the day, dyspnoea became worse, the patient became confused and $\mathrm{O}_{2}$ saturation gradually dropped to $60 \%$ on air.

Blood gas analysis showed $\mathrm{PH}-7.44, \mathrm{SPO}_{2}-90 \%$, $\mathrm{HCO}^{-}-20.6$. Initial total white cell count was 11900 $/ \mathrm{mm}^{3}$, Haemoglobin was $52 \mathrm{~g} / \mathrm{L}$, platelet count was $87,000 / \mathrm{mm}^{3}$, blood picture; features suggestive of acute blood loss. INR was 2.1, blood urea was 57 $\mathrm{mg} / \mathrm{dL}$, SGPT was $96 \mathrm{IU} / \mathrm{L}$ and SGOT was $203 \mathrm{IU} / \mathrm{L}$. Ultrasound scan of the abdomen showed mild to moderate amount of free fluid and bilateral basal pleural effusions. He was transfused with six units of blood, adequate amount of intravenous fluid and fresh frozen plasma. Oxygen saturation improved, blood pressure picked up and symptoms gradually improved. The following day we noticed an ecchymotic patch over right iliac fossa region and the patient was keeping the right hip in flexed position. $\mathrm{X}$-ray abdomen showed bulging of right psoas shadow (Figure 1).

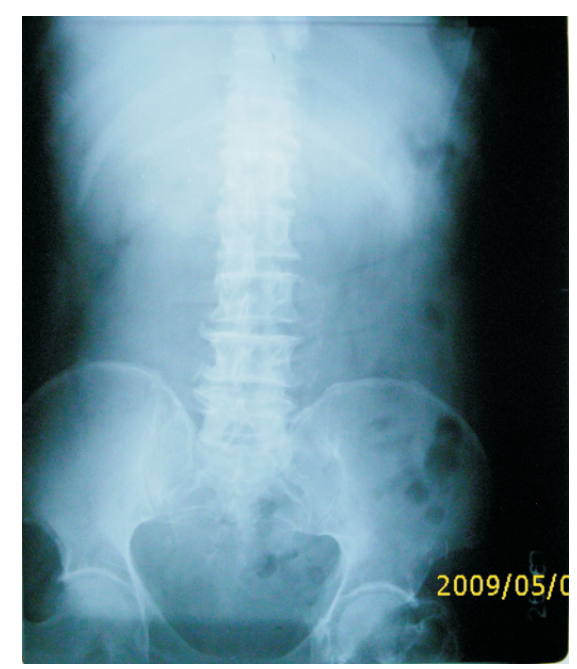

Figure 1 - X-Ray Abdomen showing bulging of right Psoas shadow 
Computer tomography of abdomen and pelvis revealed an enlargement of the right psoas muscle with heterogeneous enhancement and area of low attenuation most likely a psoas haematoma (Figure 2) and bilateral small pleural effusion with minimal ascites. On the $8^{\text {th }}$ day Dengue antibody, both IgM and $\mathrm{IgG}$, were positive. He recovered completely after two weeks.

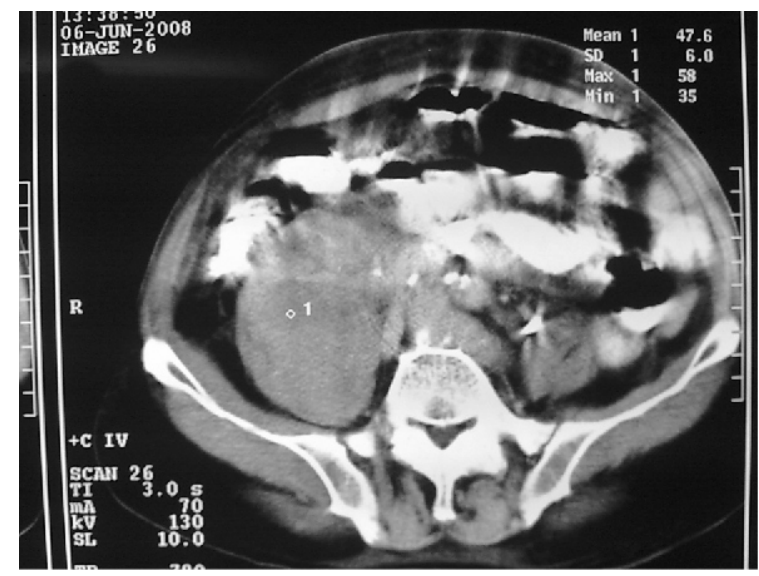

Figure 2 - CT scan of pelvis showing enlarged right psoas muscle

\section{Discussion}

The clinical course of DHF is rather stereotypic. A second infection with a different sero-type can lead to DHF with Dengue shock syndrome. The pathogenesis of bleeding is poorly understood but it is suggested that the enhanced immune response of the host to a secondary Dengue virus infection causing vasculopathy, thrombopathy and disseminated intravascular coagulation (DIC).Vasculopathy include endothelial dysfunction and increased vascular permeability. Thrombopathy consists of thrombocytopenia and platelet dysfunction. DIC is prominent in patients with shock $^{1,5}$. The illness usually begins abruptly and has three phases, namely Febrile, Critical and Convalescence. In the acute febrile phase patient has sudden rise in temperature accompanied by facial flushing, skin erythema, headache and muscle pain.
Critical phase, which lasts about $24-48$ hours during which plasma leakage, severe bleeding occurs and it coincides or follows defervescence (becoming afebrile). Varying degrees of circulatory disturbances may develop and very close monitoring of the patient is necessary at this stage ${ }^{2}$.

Compared with non-elderly adults, elderly individuals had significantly higher frequencies of concurrent bacteremia, gastrointestinal bleeding, acute renal failure and pleural effusion; higher incidence of prolonged prothrombin time and lower mean hemoglobin level ${ }^{3}$. Therefore, it is important to be aware of the protean manifestation of the disease at all times, because meticulous monitoring and appropriate treatment at the correct time can save lives.

\section{References}

1. Mathew A, Rothman AL. Understanding the contribution of cellular immunity to Dengue disease pathogenesis. Immunological Review 2008 october;225;300-13

2. Guidelines on clinical management of Dengue fever / Dengue Haemorrhagic Fever 2005.

3. Lee IK, Liu JW, YangKD. Clinical and Laboratory characteristics and risk factor for fatality in elderly patients with dengue haemorrhagic fever. American Journal of Tropical Medicine and Hygiene 2008 August; 79(2): 149-53.

4. Dengue haemorrhagic fever: diagnosis, treatment, prevention and control. $2^{\text {nd }}$ edition. Geneva: World Health Organization 1997.

5. Srichaikul T, Nimmannitya S. Haematology in Dengue and Dengue Haemorrhagic Fever; Bailliere's Best Practice and Research Clinical Haematology Jun 2000; 13(2): 261-76. 